\title{
Workshop sobre Interação e Pesquisa de Usuários no Desenvolvimento de Jogos (WIPlay)
}

\author{
Ticianne Darin \\ Instituto Universidade Virtual \\ Universidade Federal do Ceará \\ Fortaleza, Brasil \\ ticianne@virtual.ufc.br
}

\author{
Thiago Barcelos \\ Laboratório de Computação Aplicada - LABCOM ${ }^{3}$ \\ Instituto Federal de São Paulo \\ Guarulhos, Brasil \\ tsbarcelos@ifsp.edu.br
}

\begin{abstract}
RESUMO
Propomos o Workshop sobre Interação e Pesquisa de Usuários no Desenvolvimento de Jogos (WIPlay), um workshop interdisciplinar que visa reunir as comunidades de desenvolvimento de jogos e de Interação Humano-Computador (IHC). Ele é destinado a pesquisadores e profissionais interessados nos diversos aspectos da Interação JogadorComputador. O principal objetivo do workshop é fomentar a discussão sobre a pesquisa em fatores humanos no desenvolvimento e avaliação de jogos no cenário nacional, além de destacar os trabalhos dessa crescente área no Simpósio Brasileiro sobre Fatores Humanos em Sistemas Computacionais (IHCBr). WIPlay seguirá um formato dinâmico, envolvendo apresentações de artigos, sessões práticas de intercâmbio de experiências entre academia e indústria e mesas redondas.
\end{abstract}

\section{PALAVRAS-CHAVE}

Jogos Digitais, Design, Avaliação, Games User Research

\section{Introdução}

Em 2016, pesquisadores analisaram como as publicações de IHC no Brasil, no período de 2013 a 2015, abordavam os desafios elencados no GranDIHC-BR [14] para o período de 2012 a 2022, identificando que poucas pesquisas discutiam Valores Humanos (Desafio 4) no projeto e avaliação de soluções interativas. Posteriormente, Leitão et al. (2017) provocaram uma reflexão sobre o que nossa comunidade tem feito para avançar no escopo desse desafio, além de advogarem pela articulação de questões acerca de valores humanos por meio de uma agenda de pesquisa com ênfase na ética e na interdisciplinaridade [13]. Dentre os caminhos apontados pelos autores estão o estímulo à participação sistemática de profissionais de outras áreas nas pesquisas de IHC, além da objeção à abordagem simplificada em torno de fatores humanos isolados. Para isso, faz-se necessário promover reflexões em nossa comunidade sobre as dimensões Permission to reproduce or distribute, in whole or in part, material extracted from this work, verbatim, adapted or remixed, as well as the creation or production from the content of such work, is granted without fee for noncommercial use, provided that the original work is properly credited. IHC 2019 WORKSHOPS, Outubro 21-25, 2019, Vitória, Brasil. In Anais Estendidos do XVIII Simpósio Brasileiro sobre Fatores Humanos em Sistemas Computacionais. Porto Alegre: SBC (C) 2019 by the author(s), in accordance with the terms of the Creative Commons Attribution-NonCommercial 4.0 International Public License (CC BY-NC 4.0). individual, cultural e social dos diversos tipos de sistemas computacionais desenvolvidos, bem como a ampliação destas discussões para outras esferas.

Um campo intrinsecamente interdisciplinar do desenvolvimento de sistemas computacionais que tem crescido nos últimos anos é o de jogos digitais, os quais são uma estratégia atrativa para diversos propósitos, tais como entretenimento $[4,10]$, educação $[3,12]$ e saúde [16,11] por oferecerem acesso a mundos imersivos com inúmeras possibilidades de interação. O interesse crescente da comunidade internacional de Interação Humano-Computador no desenvolvimento e pesquisa em jogos tem sido demonstrado pelo surgimento de grupos de pesquisa como o HCI Games Group ${ }^{1}$; pela criação de trilhas dedicadas à IHC em conferências de jogos, como na IEEE Conference on Games²; e até pela criação de conferências específicas para esse tema, como o $\mathrm{CHI} \mathrm{Play}^{3}$. No Brasil, apesar do Simpósio Brasileiro de Jogos e Entretenimento Digital (SBGames) ser correlacionado ao $\mathrm{IHCBr}$ e consistir no maior evento acadêmico da América Latina na área de jogos, seu foco não está em destacar a reflexão transversal das diferentes dimensões dos fatores humanos no desenvolvimento e avaliação de jogos digitais. No entanto, nos últimos anos, a comunidade do $\mathrm{IHCBr}$ tem explorado facetas e características humanas no design e avaliação de jogos e aplicações para entretenimento digital, sob diversas perspectivas $[1,8,17]$.

Nesse contexto, identificamos a oportunidade de fomentar a discussão e troca de experiências sobre as várias dimensões dos fatores humanos em jogos digitais no cenário nacional, trazendo a perspectiva dos pesquisadores e profissionais de IHC e áreas correlatas para o contexto específico do desenvolvimento de jogos brasileiro, predominantemente composto por empresas indie. Para isso, propomos o Workshop sobre Interação e Pesquisa de Usuários no Desenvolvimento de Jogos (WIPlay), que propõe reunir as comunidades de pesquisa e desenvolvimento de jogos e IHC em torno de discussões interdisciplinares sobre suas respectivas contribuições, fronteiras e possibilidades na área. Assim, o WIPlay tenciona atender a três objetivos: proporcionar maior enfoque na área de jogos dentro da comunidade de IHC brasileira; estimular o desenvolvimento da pesquisa sobre as dimensões individual, social e cultural do

\footnotetext{
${ }^{1}$ http://gur.hcigames.com/

${ }^{2}$ http://ieee-cog.org/topics/

${ }^{3}$ https://chiplay.acm.org/2019/
} 
ser humano no desenvolvimento e avaliação de jogos digitais; e incentivar a interdisciplinaridade ao trazer profissionais da indústria e pesquisadores da área de jogos para discutir fatores humanos no IHCBr. Como resultado, nossa expectativa é que o WIPlay seja uma ferramenta para fomentar e divulgar pesquisas significativas na área, ajudando a consolidar o entendimento e a prática de IHC no desenvolvimento de jogos no Brasil.

\section{Justificativa}

Ao longo dos anos, pesquisadores tem discutido como a evolução da pesquisa na área de jogos digitais se relaciona com diversos fatores da IHC [5]. No Brasil, há diversas pesquisas recentes abordando temas correlatos, principalmente relacionados a técnicas de interação no design de jogos [20,4,16,1] além de métodos de design e avaliação de IHC adaptados para jogos em contextos específicos [6,9,15,3,2]. Esse fato dá indícios sobre o interesse da comunidade brasileira em investigar e articular estas áreas.

Dentre os diversos temas com questões de pesquisa instigantes, podemos citar player-centred [18] e human-centred game design [19], que promovem a adaptação dos métodos e técnicas do design centrado no usuário para as quatro etapas do processo de design de jogos. Além destes, há a Game User Research (GUR) [7], um campo emergente nascido de workshops desenvolvidos na ACM annual conference on Human Factors in Computing Systems (CHI), que une IHC, desenvolvimento de jogos e psicologia experimental, investigando especificamente a interação entre jogadores e jogos. Destacamos ainda o termo Player-Computer Interaction [5], que tem sido utilizado para descrever um subdomínio de pesquisa da IHC, focado nos diversos aspectos da interação humana ao jogar, incluindo interface, usabilidade, jogabilidade, experiência, interação social, cooperação, comportamento do jogador, dentre outros.

O WIPlay será o primeiro de seu tipo no $\mathrm{IHCBr}$, vinculando a pesquisa de IHC e de jogos digitais no contexto brasileiro. Workshops deste tipo, entretanto, têm sido realizados em conferências de diversos países promovendo contribuições substanciais para a área, como no caso da já mencionada trilha CHI Play.

Considerando especificamente o contexto da GUR, uma busca pela presença desse termo no texto completo de artigos indexados no repositório da ACM indica que o tema é presente desde 2012 em artigos do $\mathrm{CHI}$, considerando a trilha principal e os Extended Abstracts. Nos anais do CHI Play, a mesma busca indica que trabalhos envolvendo a GUR estão presentes em todas as edições do simpósio, iniciado em 2014. Os dados são apresentados a seguir na Tabela 1 .
Tabela 1. Artigos contendo a palavra-chave "Game User Research” em edições do CHI e CHI Play.

\begin{tabular}{|l|c|c|c|c|c|c|c|}
\hline & 2012 & 2013 & 2014 & 2015 & 2016 & 2017 & 2018 \\
\hline CHI Proceedings & 3 & 5 & 5 & 2 & 4 & 2 & 2 \\
\hline CHI Play & N/A & N/A & 6 & 5 & 2 & 5 & 3 \\
\hline
\end{tabular}

De uma forma mais abrangente, o tema de avaliação de jogos digitais tem estado presente no $\mathrm{CHI}$ de forma consistente ao longo dos anos. Uma busca por artigos que apresentem de forma conjunta o termo "game" no título e o termo "game evaluation" no resumo resulta em 98 artigos, sendo o mais antigo publicado em 2001. Desses trabalhos, 28 foram publicados entre 2016 e 2018. Outro indício pode ser identificado em uma busca nos anais da International Conference on the Foundations of Digital Games (FDG), onde o termo "Human Computer Interaction" é citado em 63 artigos desde 2009, sendo que 21 dos artigos que mencionam o termo foram publicados nos anais da edição de 2017. Ainda, em conferências IEEE como Symposium on Computational Intelligence and Games (CIG), IEEE International Games Innovation Conference (IGIC) e IEEE Games Entertainment Media Conference (GEM) mais de 200 artigos foram publicados nos últimos 10 anos analisando fatores humanos no design e avaliação de jogos, envolvendo as áreas de Games User Research e Player-Computer Interaction.

$\mathrm{Na}$ comunidade brasileira, o número de artigos publicados no $\mathrm{IHCBr}$ analisando aspectos da interação em jogos ou avaliação de jogos indica um certo protagonismo do simpósio nas discussões nessa temática: uma busca manual nos anais permite identificar 33 artigos pertinentes ao tema publicados desde 2010. Por outro lado, o SBGames recentemente não vem dando destaque a essa temática; em busca manual nos anais de 2017 e 2018, foram encontrados apenas 8 artigos relacionados, que pode ser considerado um número muito baixo considerando a quantidade total de publicações naquele simpósio.

\section{Formato}

O WIPlay é proposto como um workshop de um dia, abordando temas relacionados a métodos de design de jogos digitais centrados no ser humano, técnicas de interação em jogos digitais, além de métodos de avaliação e de pesquisa com o usuário neste contexto. A escolha de tais temas é decorrente das questões atualmente em aberto nessa área, que tem focado em ampliar e modificar as metodologias existentes usadas pela comunidade de IHC para o ambiente dos jogos digitais.

O workshop seguirá um formato dinâmico, composto por três momentos principais. Primeiro, os participantes serão convidados a apresentar uma visão geral de seus trabalhos, divididos em categorias temáticas que poderão abranger desde contribuições técnicas em novas interfaces de usuário até metodologias experimentais no desenvolvimento de jogos. Após isso, os participantes colaborarão em sessões práticas para 
explorar questões sobre como lidar com fatores humanos no desenvolvimento e avaliação de jogos, conciliando metodologias acadêmicas e orientadas à prática. O WIPlay se encerrará com uma mesa redonda onde representantes da indústria e academia serão convidados a compartilhar suas experiências e discutir com os participantes aquelas questões previamente exploradas.

O resultado das discussões do workshop será submetido ao IHC 2020, relatando a coleção de lições aprendidas a partir da prática do design e avaliação de jogos no contexto brasileiro e um apanhado das técnicas comumente utilizadas. Isso ampliará a discussão dos tópicos para além do workshop e servirá como uma plataforma para o desenvolvimento de trabalhos futuros.

\section{Tópicos de Interesse}

Os autores serão convidados a enviar trabalhos originais que apresentem contribuições articulando as áreas de IHC e jogos digitais, focando em avanços na compreensão da experiência do jogador, bem como contribuições em projetos inovadores ou implementações de interações jogador-computador, incluindo, mas não limitado, aos seguintes temas:

1. Análise e discussão de tendências na pesquisa sobre interação com jogos e aplicações de entretenimento;

2. Técnicas de implementação inovadoras que afetam as experiências dos jogadores;

3. Métodos e técnicas para avaliação da experiência e comportamento do jogador (gameplay experience, game experience, player experience, dentre outros);

4. Design e avaliação de jogos acessíveis e inclusivos;

5. Psicologia e comportamento dos jogadores;

6. Games User Research no desenvolvimento e avaliação de jogos e aplicações de entretenimento;

7. Estudos de caso e adaptações de métodos e técnicas da IHC para Human-Centred e Player-Centered Game Design

8. Game analytics e novas visualizações das experiências dos jogadores

9. Fatores Humanos (individual, cultural e social) no desenvolvimento de jogos para contextos específicos

10. Estudos de caso e lições aprendidas de profissionais e pesquisadores na indústria de desenvolvimento de jogos, incluindo gamificação, pesquisa de usuários de jogos e lacunas entre a pesquisa acadêmica e a prática aplicada.

\section{Divulgação e Processo de Seleção}

Considerando as características interdisciplinares da temática proposta para o WIPlay, a divulgação será feita, inicialmente, junto às comunidades de IHC, Jogos Digitais e Informática na Educação. Ainda, considerando a importância do tema para o fortalecimento da indústria nacional, será feita também a divulgação em fóruns relacionados a desenvolvedores e cursos universitários com disciplinas específicas sobre design de jogos. Ainda, visando garantir a qualidade dos trabalhos a serem selecionados, já foram iniciadas sondagens preliminares junto a pesquisadores experientes na comunidade de IHC envolvidos com a pesquisa em jogos digitais para a composição do futuro
Comitê de Programa. Também será uma meta da organização do WIPlay convidar pesquisadores com vínculo com a indústria para o Comitê de Programa de forma a estabelecer esse desejável intercâmbio.

Durante o processo de seleção, cada submissão será revisada por pelo menos três pesquisadores em IHC com experiência em jogos. Os artigos serão selecionados para publicação e apresentação com base nos seguintes critérios: qualidade do trabalho, relevância da contribuição, adequação aos temas propostos e potencial do trabalho para suscitar discussões construtivas durante o workshop.

Os autores dos melhores artigos serão convidados a submeter versões estendidas do trabalho a periódicos relacionados aos tópicos de interesse do WIPlay. No momento da submissão desta proposta, os organizadores já tem definida a possibilidade de submissão à Revista Sistemas e Mídias Digitais (ISSN 2525-9555), da Universidade Federal do Ceará, enquanto que outros espaços para publicação estão sendo negociados.

\section{Currículo Resumido dos Organizadores}

\subsection{Ticianne de Gois Ribeiro Darin}

Doutora em Ciência da Computação (2018) pela Universidade Federal do Ceará, com período sanduíche na Universidad de Chile. No doutorado, investigou a avaliação de usabilidade e impacto cognitivo de jogos multimodais projetados para desenvolver habilidades em pessoas cegas. Durante esse período, participou de três projetos que envolveram o desenvolvimento e avaliação de jogos nesse contexto. Desde 2011, é professora da Universidade Federal do Ceará, onde também é pesquisadora do Grupo de Redes de Computadores, Engenharia de Software e Sistemas (GREat) e coordena o grupo de estudos e pesquisa Célula de Design e Multimídia. Atualmente, é professora adjunta da Universidade Federal do Ceará.

\subsection{Thiago Schumacher Barcelos}

Doutor em Ensino de Ciências e Matemática (2014) pela Universidade Cruzeiro do Sul, onde pesquisou a construção de jogos digitais como estratégia didática para o ensino de Computação e Matemática na Educação Básica, bem como o impacto da jogabilidade dos jogos construídos como fator motivacional. Desde 2008 é professor e pesquisador no Instituto Federal de Educação, Ciência e Tecnologia de São Paulo, onde lidera o grupo de pesquisa $\mathrm{LABCOM}^{3}$ - Laboratório de Computação Aplicada e o IFGAMES - Núcleo de Estudos e Desenvolvimento de Games do IFSP Guarulhos.

\section{Cronograma Proposto}

Os organizadores, em caso de aprovação da proposta deste workshop, se comprometem a cumprir o cronograma definido abaixo. Eventos de força maior ou determinações da organização geral do IHC serão considerados e podem influenciar os prazos abaixo, sem prejuízo da qualidade que se espera para o evento. 
- Definição do Comitê de Programa: Até 30/06/2019

- Data inicial para divulgação da chamada de artigos: $10 / 06 / 2019$

- Período de submissão de artigos: 12/06/2019 a 29/07/2019

- Prazo limite para avaliação pelo Comitê de Programa: $02 / 09 / 2019$

- $\quad$ Notificação de aceitação: 06/09/2019

- Envio da versão final pelos autores (camera-ready): $20 / 09 / 2019$

\section{REFERÊNCIAS}

[1] Pablo Eduardo Cabral Barata and Marcelle Pereira Mota. 2018. A Proposal for an Adaptive Game to Support the Skills Development in Children with Disabilities. In Proceedings of the 17th Brazilian Symposium on Human Factors

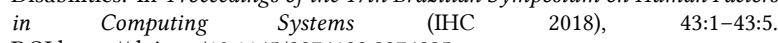
DOI:https://doi.org/10.1145/3274192.3274235

[2] Thiago S. Barcelos, Roberto Muñoz, and Virgínia Chalegre. 2012. Gamers As Usability Evaluators: A Study in the Domain of Virtual Worlds. In Proceedings of the 11th Brazilian Symposium on Human Factors in Computing Systems (IHC '12), 301-304. Retrieved from http://dl.acm.org/citation.cfm?id=2393536.2393580

[3] Clodis Boscarioli, Charles Giovane de Salles, Jeferson José Baqueta, and João Paulo Colling. 2012. Avaliação e Design De Interação De Jogos Voltados Ao Aprendizado De Crianças Surdas. In Companion Proceedings of the 11th Brazilian Symposium on Human Factors in Computing Systems (IHC '12), 2526. Retrieved from http://dl.acm.org/citation.cfm?id=2400076.2400086

[4] Rafael Cunha Cardoso, Vinícius Kruger da Costa, Andreia Sias Rodrigues, Tatiana Aires Tavares, Krishna Ferreira Xavier, Jamir Alves Peroba, Juliana Peglow, and CLSM Quadros. 2016. Doce labirinto: Experiencia de jogo utilizando interação baseada em movimentos da cabeça e recursos tangíveis. In XV Simpósio Brasileiro de fogos e Entretenimento Digital.

[5] Marcus Carter, John Downs, Bjorn Nansen, Mitchell Harrop, and Martin Gibbs. 2014. Paradigms of Games Research in HCI: A Review of 10 Years of Research at CHI. In Proceedings of the First ACM SIGCHI Annual Symposium on Computer-human Interaction in Play (CHI PLAY '14), 27-36. DOI:https://doi.org/10.1145/2658537.2658708

[6] Ticianne Darin, Rossana Andrade, and Jaime Sánchez. 2018. SLUP: A Standard List of Usability Problems in Multimodal Video Games designed for People Who Are Blind. In Anais Estendidos do XVII Simpósio Brasileiro sobre Fatores Humanos em Sistemas Computacionais. DOI:https://doi.org/10.5753/ihc.2018.4231

[7] Anders Drachen, Pejman Mirza-Babaei, and Lennart E Nacke. 2018. Games user research. Oxford University Press.

[8] Emanuel Felipe Duarte and M. Cecília C. Baranauskas. 2018. Revisiting Interactive Art from an Interaction Design Perspective: Opening a Research Agenda. In Proceedings of the 17th Brazilian Symposium on Human Factors in $\begin{array}{llll}\text { Computing } & \text { Systems (IHC 2018), 35:1-35:10. }\end{array}$ DOI:https://doi.org/10.1145/3274192.3274227

[9] Flávia Santos e André Salgado e Renata Fortes. 2018. Um Mapeamento Sistemático sobre Acessibilidade e Usabilidade no Desenvolvimento de Jogos Digitais para Idosos. ISys - Rev. Bras. Sist. Informação 11, 2 (2018), 63-90.

[10] Trisha Garcia, Shannon Brown, and Siona Dev. 2018. TUIst: A Collaborative and Computationally Enhanced Game Board. In Proceedings of the Twelfth International Conference on Tangible, Embedded, and Embodied Interaction (TEI '18), 337-342. DOI:https://doi.org/10.1145/3173225.3173282

[11] M. Ghassemi, K. Triandafilou, A. Barry, M. E Stoykov, E. Roth, F. A. MussaIvaldi, D. G. Kamper, and R. Ranganathan. 2019. Development of an EMGControlled Serious Game for Rehabilitation. IEEE Trans. Neural Syst. Rehabil. $\begin{array}{llll}\text { Eng. } & 27, & 2 & \text { (February 2019), 283-292. }\end{array}$ DOI:https://doi.org/10.1109/TNSRE.2019.2894102

[12] Y. J. Lan, I. Y. Hsiao, and M. F. Shih. 2018. Effective Learning Design of Game-Based 3D Virtual Language Learning Environments for Special Education Students. 7. Educ. Technol. Soc. 21, 3 (2018).

[13] Carla Leitão, Cristiano Maciel, Lara Schibelsky Godoy Piccolo, Luciana Salgado, Patricia C. de Souza, Raquel Prates, Roberto Pereira, and Vinicius Carvalho Pereira. 2017. Human Values in HCI: A Challenge for the GrandIHC-BR. In Proceedings of the XVI Brazilian Symposium on Human Factors in Computing Systems (IHC 2017), 70:1-70:6. DOI:https://doi.org/10.1145/3160504.3160565

[14] Andre de Oliveira Bueno, Lucas Cesar Ferreira, Vinicius Ferreira, and Junia Coutinho Anacleto. 2016. Research Trends in HCI in Brazil: An Analysis in Relation to the GranDIHC-Br. In Proceedings of the 15th Brazilian Symposium on Human Factors in Computing Systems (IHC '16), 22:1-22:10. DOI:https://doi.org/10.1145/3033701.3033723
[15] Juan Oliveira and Wilson Prata. 2018. Pesquisa de UX em jogos de Realidade Virtual: desafios e lições aprendidas. In Anais Estendidos do XVII Simpósio Brasileiro sobre Fatores Humanos em Sistemas Computacionais. DOI:https://doi.org/10.5753/ihc.2018.4180

[16] Ariane Rodrigues, Hérikles Cordeiro, José Anderson Soares da Silva, Ramon Silva, and Bruno Barboza. 2018. Batalha do Sorriso: Processo de desenvolvimento de um jogo sério para a Saúde Bucal. In Anais dos Workshops do VII Congresso Brasileiro de Informática na Educação (WCBIE 2018).

[17] Danilo Barros dos Santos, Cristiano Maciel, Vinicius Carvalho Pereira, and Eunice Pereira dos Santos Nunes. 2018. Analysis of The Perception of Users of Empathic Games in Discussion Forums and Their Relation to Death. In Proceedings of the 17th Brazilian Symposium on Human Factors in Computing Systems (IHC 2018), 6:1-6:10. DOI:https://doi.org/10.1145/3274192.3274198

[18] Jonathan Sykes and Melissa Federoff. 2006. Player-centred Game Design. In CHI '06 Extended Abstracts on Human Factors in Computing Systems (CHI EA '06), 1731-1734. DOI:https://doi.org/10.1145/1125451.1125774

[19] Veronika A. Vanden Abeele and Veerle Van Rompaey. 2006. Introducing Human-centered Research to Game Design: Designing Game Concepts for and with Senior Citizens. In CHI '06 Extended Abstracts on Human Factors in Computing Systems (CHI EA '06), 1469-1474. DOI:https://doi.org/10.1145/1125451.1125721

[20] Christiane Gresse von Wangenheim, Djone Kochanski, and Rafael Savi. 2009. Revisão sistemática sobre avaliação de jogos voltados para aprendizagem de Engenharia de Software no Brasil. In Anais do $2^{\circ}$ Fórum de Educação em Engenharia de Software. 